\title{
Pendulum Experiments for a Vibration Laboratory
}

\author{
Frank M. Kelso, Ph.D. \\ Mechanical Engineering Department \\ University of Minnesota
}

\begin{abstract}
An introductory course in vibration engineering naturally begins with the basic "building block" concepts on which a deeper understanding is built. The study of single degree of freedom lumped parameter systems lays the conceptual groundwork needed for studying the behavior of multidegree of freedom or continuous systems. The simplest single degree of freedom vibratory system is of course the pendulum. Pendulum problems illustrate basic vibration theory very nicely, providing a simple and intuitive illustration of the building block concepts: lumped parameter models, simple harmonic motion, natural frequency, damped and undamped systems, free and forced response. Constructing these simple pendulum arrangements in a "testbed" environment and measuring the actual natural frequency provides a useful vehicle for extending theory into actual practice. This paper examines one particular pendulum problem and its implementation and use in a laboratory setting.
\end{abstract}




\section{Background}

The vibration lab is intended to provide students with an introduction to the theory of mechanical vibrations, and the practice of identifying and measuring vibrations in actual systems.

Vibration theory covered in this lab begins with the basic lumped parameters used to model simple mechanical systems: mass, damping, and structural stiffness. These building blocks are used to construct single degree of freedom (SDOF) analytical models of mechanical systems, which may then be used to predict vibration characteristics such as natural frequency.

Vibration practice in the laboratory involves instrumenting an actual system and determining actual vibration characteristics (e.g. natural frequency.) In addition to learning how to use transducers and data acquisition systems to collect and analyze vibration data, the student will (1) gain experience and confidence in the use of simple models, and (more importantly) (2) gain experience in discovering the source and magnitude of discrepancies between ideal analytical models and actual physical systems.

Students spend a great deal of time developing and using analytical models during the course of their undergraduate curriculum. A lab class can provide a valuable reminder of the limitations and approximations inherent in these analytical models.

\section{Illustrative Example}

A typical textbook problem taken from Steidel [1] is shown in Figure 1 below:

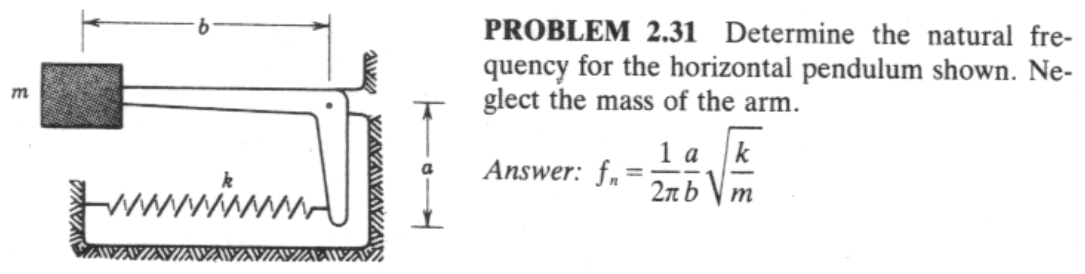

Figure 1: Problem 2.31 from Steidel 
Solving this textbook problem provides an excellent application of vibration fundamentals. The student constructs a free body diagram of a lumped parameter model of this system (Figure 2 below), then performs a torque balance to develop the system model:

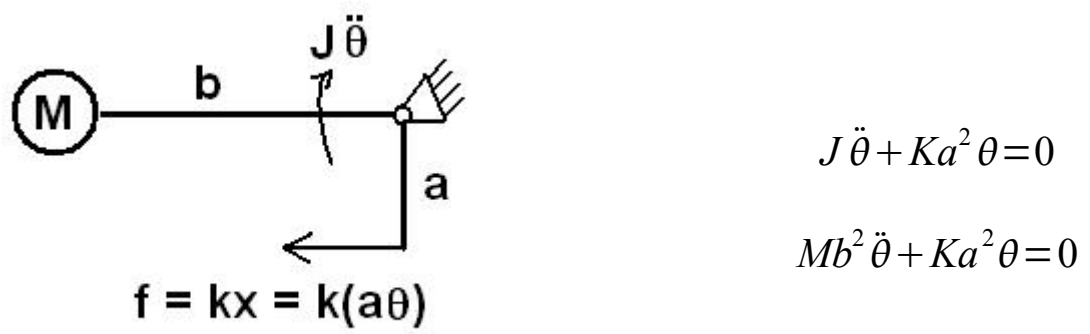

Figure 2: Free Body Diagram

This model can then be used to calculate the (ideal) natural frequency of the system:

$$
w_{n}^{2}=\frac{a^{2}}{b^{2}} \times \frac{K}{M} \quad \mathrm{rad}^{2} / \mathrm{sec}^{2}
$$

This simple analytical model is based on the assumption that (1) friction is insignificant, and (2) distributed quantities such as mass and compliance may be treated as lumped parameters. Theory can be connected to the real world using a physical implementation of this SDOF pendulum.

\section{Pendulum Construction}

The pendulum of Figures 1 and 2 is constructed from a (modified) 12-inch steel utility square (Figure 3 below). 


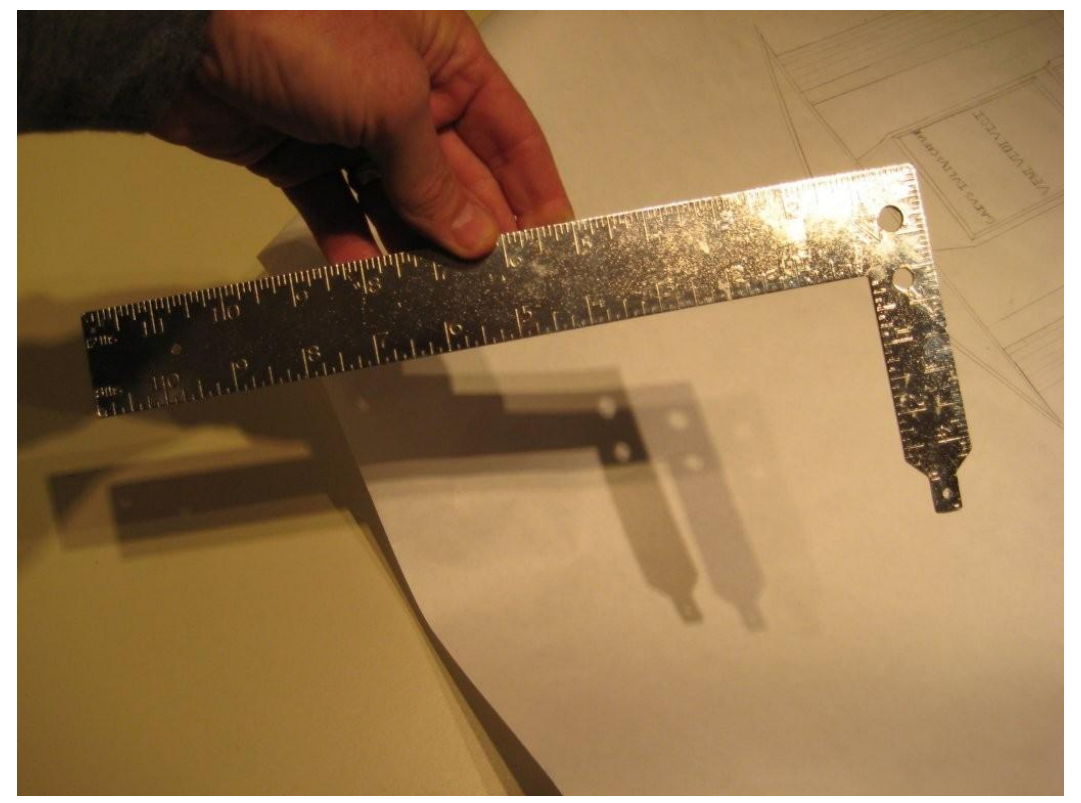

Figure 3: Utility Square (Customized)

An adjustable weight is mounted on the top of the square, and its horizontal position can be adjusted by sliding it into place and locking it down with a set screw. The adjustable weight assembly is shown below in Figure 4. The top of the weight assembly is drilled and tapped to accommodate an accelerometer.

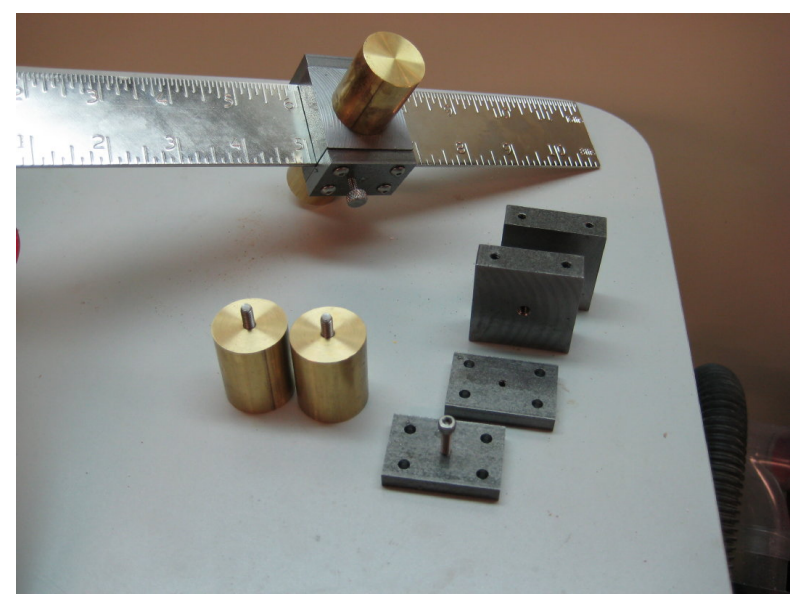

Figure 4: Adjustable Weight Assembly 
A hole to receive the axle is shown at the junction of the long and short legs of the L-Square (Figure 3), and a hole to accommodate spring(s) is shown at the tapered end of the short leg. A photo of the pendulum and its support plate is shown in Figure 5 below.

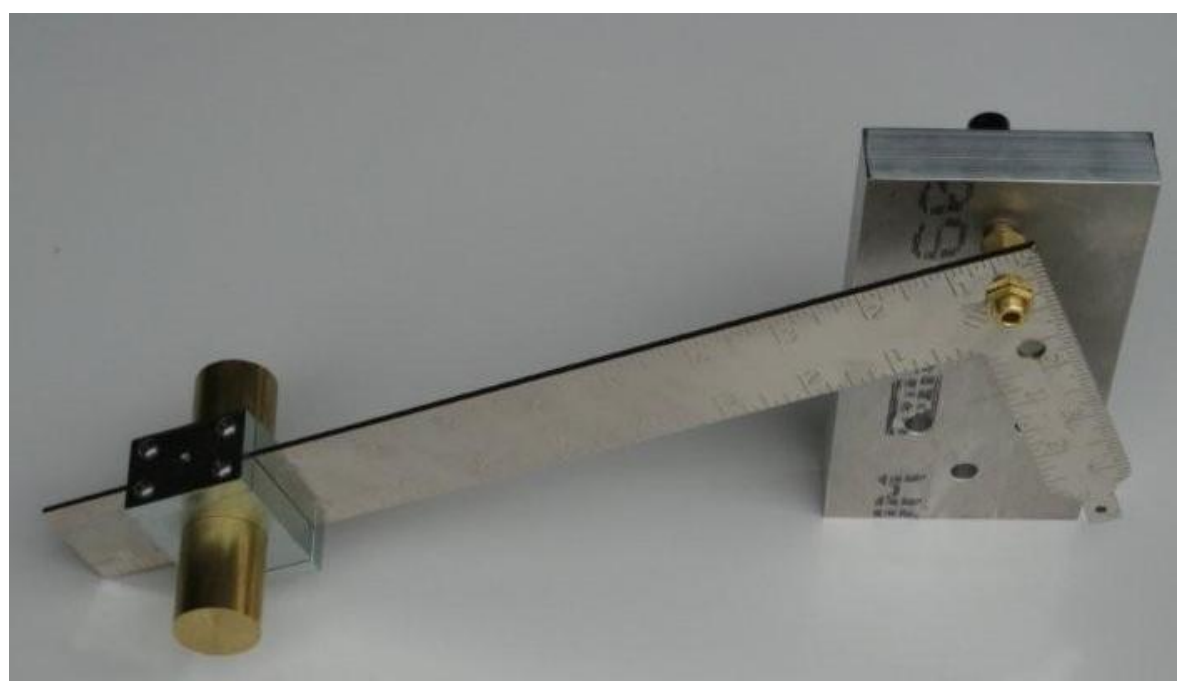

Figure 5: Physical Pendulum, Axle and Support Plate

The support plate serves two purposes. First, it supports the axle on which the pendulum oscillates (Figure 5, Figure 6B). Second, it allows a potentiometer to be attached to the pendulum axle for measuring and recording angular position. This is shown in Figure 6A below.

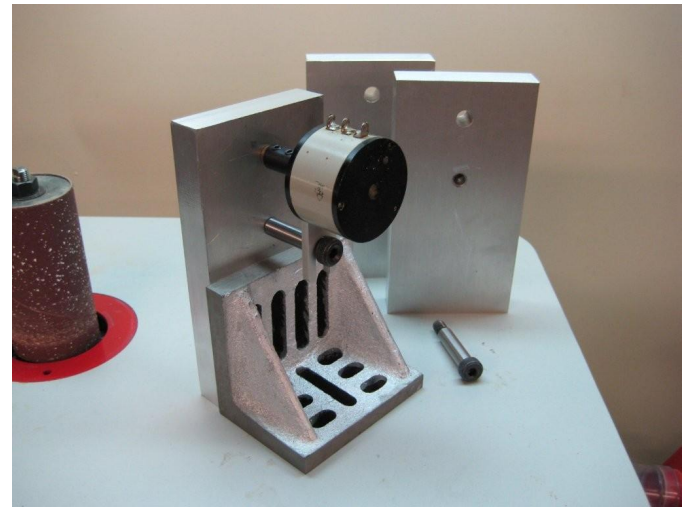

Figure 6A: Mounting the Potentiometer

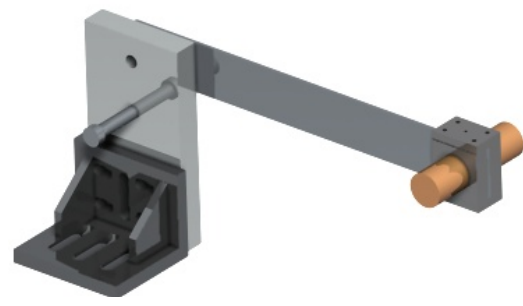

Figure 6B: Supporting the Pendulum Axle 
The support plate assembly is fixed to ground by providing it with T-bolts which attach to a TSlot baseplate, shown in Figure 7 below. The aluminum baseplate supplies a reaction mass to the oscillating pendulum.

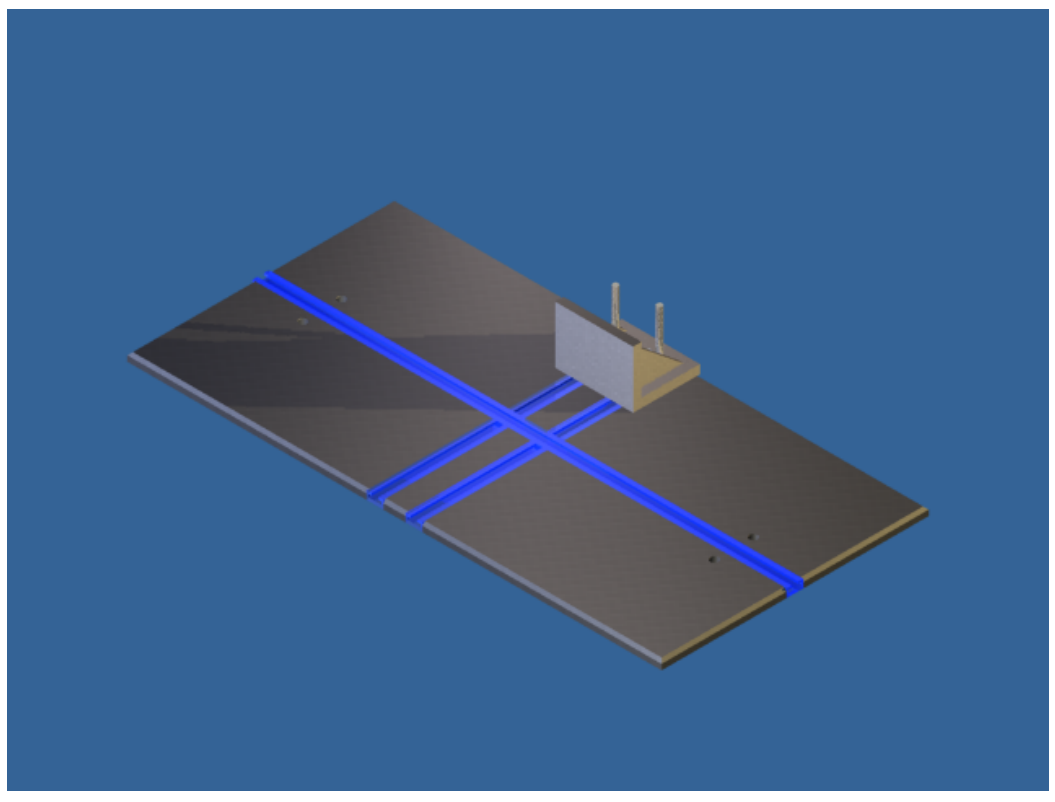

Figure 7: T-Slot Base Plate (Rendering)

Adding mill feet to the angle bracket allows the pendulum to be positioned along the double TTrack arrangement, as shown in Figure 7. Once positioned with the pendulum centered over the central T-Slot, the pendulum assembly is locked in place using T-bolts and cam clamps. The single T-Track down the center is used to accommodate a similar adjustable bracket and spring. The spring is attached to the short leg of the pendulum and tensioned by sliding the spring bracket along the single track and clamping it in place. Once the long leg of the pendulum is lifted parallel to the surface of the baseplate, the spring is clamped in place and the pendulum is free to oscillate about its static equilibrium position. 
Given an initial displacement, the pendulum will oscillate at its natural frequency. The potentiometer will record the angular position as a function of time, and both the damping ratio $\xi$ and the damped natural frequency $\omega_{\mathrm{D}}$ may be calculated from the data.

\section{Theory and Practice}

There are always discrepancies between idealized analytical models and actual physical systems, and engineering students must learn to reconcile these discrepancies. On paper the natural frequency of the pendulum system shown in Figure 1 is calculated as $36 \mathrm{rad} / \mathrm{sec}(5.7 \mathrm{~Hz})$. In fact, the measured frequency is $33.4 \mathrm{rad} / \mathrm{sec}(5.3 \mathrm{~Hz})$. The student is therefore faced with important questions that don't typically come up in a textbook problem but do typically come up in engineering practice: questions such as:

- Is the discrepancy significant?

- Can it be accounted for by the simplifying assumptions that were made in the calculation?

- Can it be accounted for in the measurement of the system parameters ( $\mathrm{K}$ and $\mathrm{M}$, as well as lengths a and b)? Would an uncertainty analysis be helpful at this point? (yes!)

- How accurate is the lumped parameter idealization of a distributed system?

- Is friction significant or may it be neglected (undamped versus damped natural frequency)?

- Where could error enter in to the measurement process?

- Where could error enter in to the data reduction process?

All of these questions prompt students to reflect more deeply on the analytical model as well as the measurement process and its limitations. This is the value of a laboratory associated with a lecture-type class: it keeps the theory grounded in reality. 


\section{Example: Rethinking the Model}

In reviewing the development of equation (1), most students immediately identified the assumption of a massless L-Square as a simplification they could easily eliminate. Several proposals were put forward for determining the rotary inertia of the L-Square and its effect on the natural frequency. The rotary inertia of this "massless" component could be determined:

- $\quad$ analytically - by hand

- analytically - using a computer

- experimentally

The hand calculation of an ideal L-shape is very straightforward, but once again an idealization creeps in. The actual shape of the pendulum is a modified $\mathrm{L}$, with drilled holes and tapered edges. Accounting for these modifications becomes tedious - much more suited to computer calculation.

Using standard engineering software such as Pro/Engineer $\AA$, SolidWorks $₫$ or Inventor $\AA$, students can draft the L-Square and then list its properties, as shown in Figure 8 below. (This was in fact a post-lab exercise.)

The rotary inertia of the pendulum could also be determined experimentally using a trifilar suspension to measure the inertia. Trifilar suspensions were covered in an earlier lab, and dovetailed nicely with the current lab. By happy coincidence this earlier lab included measuring the rotary inertia of the L-Square. The experimental results were corroborated by the computer model and the result used to correct the estimate of the (ideal) natural frequency. 


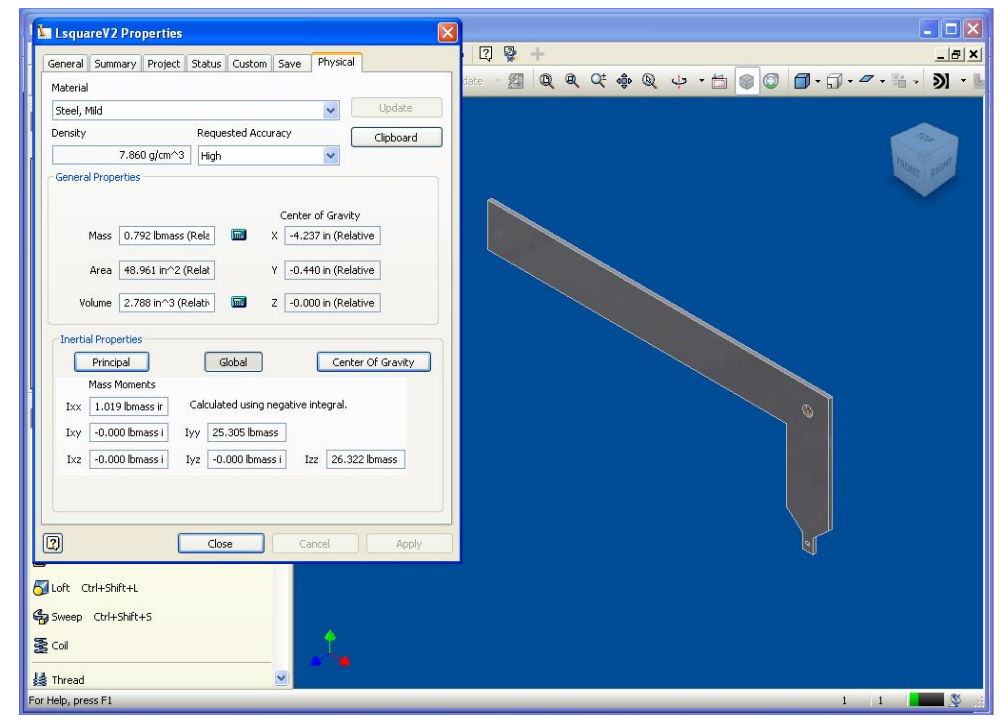

Figure 8: Rotary Inertia of the the "Massless" L-Square

\section{Conclusion}

The textbook problem in Figure 1 provided an excellent exercise for learning and reinforcing basic vibration concepts: simple harmonic motion, natural frequency, construction and use of lumped parameter system models. Connecting the textbook problem to an actual physical system raised many interesting and practical questions. These questions in turn motivated further investigation and a deeper understanding of vibration theory.

The T-Slot baseplate and fixturing facilitates the development and use of other single degree of freedom pendulum geometries. Sample problems (again taken from [1]) and future pendula are shown in the Figure 9 below.
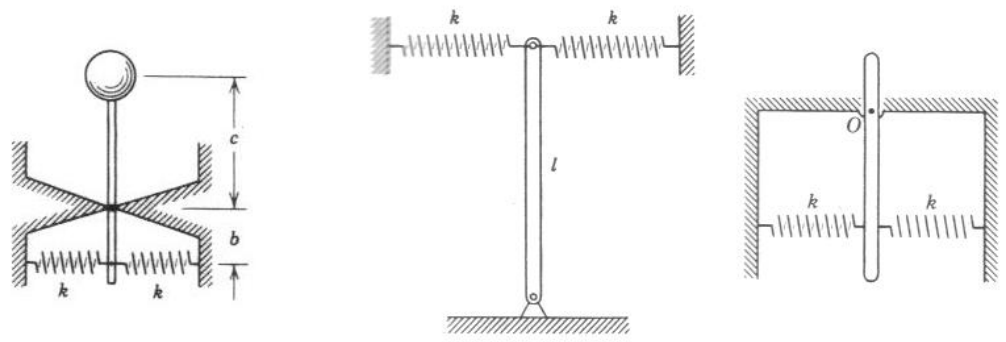

Figure 9: Other Pendulum Geometries (ref. [1]) 


\section{References}

[1] Steidel, Robert F. (1989). An Introduction to Mechanical Vibrations (3rd ed.). New York: John Wiley and Sons 